\title{
PERFIL CLÍNICO E EPIDEMIOLÓGICO DOS IDOSOS PORTADORES DO HIV/AIDS DE UMA UNIDADE DE REFERÊNCIA EM BELÉM-PA
}

\author{
Tereza Cristina dos Reis FERREIRA ${ }^{1}$ \\ Ana Paula Costa de SOUZA ${ }^{2}$ \\ Roberto Sena RODRIGUES JÚNIOR ${ }^{3}$
}

\begin{abstract}
${ }^{1 .}$ Fisioterapeuta. MSc em Saúde, Sociedade e Endemias na Amazônia (UFAM). Docente do Curso de Fisioterapia do Centro Universitário do Pará (CESUPA) e da Universidade do Estado do Pará (UEPA). tereza_reis@ yahoo.com.br ${ }^{2}$ Discentes do $8^{\circ}$ semestre do Curso de Fisioterapia do Centro Universitário do Pará (CESUPA). anapaulasouza76@yahoo.com.br robertojr27@yahoo.com.br
\end{abstract}

Recebido em: 22/12/2014 - Aprovado em: 05/08/2015 - Disponibilizado em: 30/10/2015

RESUMO: O HIV/AIDS apresenta-se como uma problemática crônica, que não distingue sexo, nível socioeconômico ou idade, acometendo, também, os idosos. O objetivo do estudo foi o de verificar o perfil clínico e epidemiológico dos idosos portadores do HIV/AIDS de uma Unidade de Referência em Belém-PA. Tratou-se de um estudo descritivoanalítico retrospectivo, utilizaram-se prontuários dos anos de 2000 a 2013. Dos 1.134 prontuários analisados, apenas 23 apresentaram no registro de admissão idade igual ou superior a 60 anos. Dos 23 prontuários selecionados para esse estudo, a maioria era do sexo masculino (82,61\%), na faixa etária de 60-69 anos $(95,65 \%)$, casados $(56,52 \%)$, com o fundamental incompleto $(39,12 \%)$, de Belém ou região metropolitana $(73,91 \%)$, não adquiriu nenhuma coinfecçãoHIV/AIDS (60,86\%), fez uso de terapia antirretroviral $(91,30 \%)$ e estava em continuidade de tratamento. Acredita-se que essa amostra seja maior do que a apresentada, em virtude das subnotificações e dos "não-diagnósticos" nos idosos. Palavras-Chave: Epidemiologia. Síndrome da Imunodeficiência Adquirida. Vírus da Imunodeficiência Humana. Idoso. Registros Médicos.

\section{CLINICAL AND EPIDEMIOLOGICAL PROFILE OF ELDERLY PATIENTS WITH HIV/AIDS OF A REFERENCE UNIT FROM BELÉM-PA}

\begin{abstract}
HIV/AIDS presents as a chronic problem, not distinguishing sex, socioeconomic status or age, affecting the elderly, too. The objective of this study was to verify the clinical and epidemiological profile of elderly patients with HIV/AIDS of a Reference Unit from Belém-PA. It was a retrospective descriptive-analytic study that used medical records of the 2000-2013 years. Of the 1,134 records medical analyzed, only 23 showed the admission record aged greater than or equal 60 years. Of the 23 selected medical records for this study, the majority was male $(82.61 \%)$, aged 60-69 years $(95.65 \%)$, married $(56.52 \%)$, with incomplete primary $(39,12 \%)$, from Belém or metropolitan area (73.91\%), no acquired coinfection HIV / AIDS (60.86\%), made use of antiretroviral therapy (91.30\%) and was in continuity of treatment. It was estimated that this sample be greater than this one presented because of underreported and "non-diagnostic" in elderly.
\end{abstract}

Keywords: Epidemiology. Acquired Immunodeficiency Syndrome. HIV. Aged. Medical Records.

\section{INTRODUÇÃO}

A Síndrome da Imunodeficiência

Adquirida (SIDA) apresenta-se como uma doença crônica, debilitante e contagiosa, levantando a necessidade de se reformular o cuidado em saúde (SOUSA; SILVA, 2013). $\mathrm{E}$, devido ao seu rápido avanço e disseminação, percebe-se que tal Síndrome acomete diferentes culturas, faixas etárias e 
níveis socioeconômicos (BERTONI et al, 2010).

Nessa perspectiva, Cambruzzi e Lara (2012), elencam que há uma tendência, em curto período tempo, que o número de idosos contaminados pelo Vírus da Imunodeficiência Humana (HIV) será aumentado significativamente, em virtude da vulnerabilidade física e psicológica, do baixo acesso a serviços de saúde e da invisibilidade com a qual é tratada a exposição de risco do idoso ao HIV/AIDS, seja por via sexual ou uso de drogas ilícitas.

O envelhecimento é tido como um processo natural, no qual o indivíduo é submetido a diversas alterações físicas e funcionais, sendo que tais alterações acontecem de forma progressiva, resultando em reduções na capacidade funcional do indivíduo (SILVEIRA et al, 2011).

De acordo com Brasil (2014), 757.042 casos de HIV/AIDS foram notificados no Brasil de 1980 até junho de 2014. Dessa quantidade, 23.271 casos foram de indivíduos com 60 anos ou mais. Em relação o acometimento da AIDS segundo o sexo na terceira idade, 14.756 casos foram do sexo masculino e 8.515 do feminino.
No Brasil, aproximadamente, 2,5\% dos idosos são portadores do HIV/AIDS. No entanto, essa porcentagem pode ser maior, em virtude da subnotificação dos casos e do "nãodiagnóstico", resultando em elevados índices de mortalidade (BRASIL, 2009; BATISTA et al, 2011).

Aliado a isso, existe o desafio de diagnosticar o HIV/AIDS precocemente em idosos, em virtude de se tratar de um diagnóstico diferencial a um grupo já exposto a diversas patologias, que podem ser confundidas com manifestações de doenças típicas nessa faixa etária (fadiga e perda ponderal), acarretando em subnotificações ou diagnósticos tardios, aumentando a probabilidade de infecções oportunistas e complicações (SERRA et al, 2013; TOLEDO et al, 2010).

De acordo com Serra et al (2013) diversas são as causas correlacionadas ao aumento de contaminação e de idosos vivendo com HIV/AIDS, dentre elas: as mudanças socioculturais, sobretudo na sexualidade; a resistência por parte dos idosos em fazer o uso da camisinha; as inovações nas áreas da saúde e medicamentosa; o acesso à terapia antirretroviral etc.

Além disso, é importante que os serviços de saúde acolham a terceira idade e 
desenvolvam ações voltadas para o HIV/AIDS, pois, sabe-se que a maioria dos idosos não busca apoio nesses serviços por vergonha de ser visto recebendo orientações sexuais ou preservativos, ou, quando já infectados, de serem descobertos por conhecidos e, consequentemente, estigmatizados e discriminados (GARCIA et al, 2012).

Diante do exposto, o presente estudo teve como objetivo avaliar o perfil clínico e epidemiológico dos idosos portadores do HIV/AIDS de uma Unidade de Referência em Belém-PA.

\section{METODOLOGIA}

Tratou-se de um estudo descritivoanalítico, de caráter retrospectivo, realizado em uma Unidade de Referência especializada em Doenças Infecciosas Parasitárias Especiais (URE DIPE), localizada na cidade de BelémPA. Dos 1.134 prontuários de indivíduos analisados, apenas 23 foram selecionados por atenderem o critério de inclusão: ter 60 anos ou mais no registro de admissão, quando diagnosticado pelo HIV/AIDS, no período de janeiro de 2000 a dezembro de 2013.

Os dados foram coletados no mês de novembro de 2014, por meio de consulta direta aos prontuários, e as informações obtidas foram transcritas para uma ficha que analisava as variáveis demográficas (sexo, faixa etária, estado civil, escolaridade e procedência) variáveis clínicas (coinfecção, uso de terapia antirretroviral e evolução do tratamento).

O banco de dados foi organizado em planilhas Microsoft Excel 10 e posteriormente processado e analisado por meio do software Statistical Package for Social Science (SPSS) versão 18.0.

O projeto foi aprovado pelo Comitê de Ética em Pesquisa do Centro Universitário do Pará (CAAE: 37550814.5.0000.5169), e respeitou os preceitos da Resolução nº 466/12 do Conselho Nacional de Saúde.

\section{RESULTADOS}

Dos $23 \quad(100 \%)$ prontuários selecionados, observou-se que $19(82,61 \%)$ eram do sexo masculino e $4(17,39 \%)$ do sexo feminino. No que se refere a faixa etária, nos homens, $18(94,74 \%)$ estavam na faixa etária de 60-69 anos e $1(5,26 \%)$ na faixa de 70-79 anos. Em relação às mulheres, a totalidade, encontrava-se no intervalo de 60-69 anos. Quanto ao estado civil, no sexo masculino, 4 $(21,05 \%)$ eram solteiros, $13(68,42 \%)$ eram casados e 2 viúvos (10,53\%). No sexo feminino, 2 eram solteiras e 2 viúvas $(50 \%$ cada) (TABELA 1). 
Na variável escolaridade, dos homens, houve prevalência do ensino fundamental incompleto $(42,11 \%)$ e, nas mulheres, não houve uma escolaridade com prevalência maior em detrimento das demais (TABELA 2).

Quanto à procedência foi observado que a maioria dos homens $(68,42 \%)$ e a totalidade das mulheres (100\%) eram de Belém ou região metropolitana. No tocante coinfecção, percebeu-se que a maioria dos indivíduos portadores do HIV/AIDS, tanto os homens $(57,91 \%)$ quanto as mulheres $(75,00 \%)$, não veio a adquirir nenhuma coinfecção oportunista paralela, sendo que, nos homens, daqueles que vieram a adquirir uma coinfecção, a tuberculose $(21,05 \%)$ se destacou. (TABELA 3).

Com relação ao uso de terapia antirretroviral (TARV), constatou-se que tanto a maior parte dos homens $(89,48 \%)$, assim como a totalidade das mulheres (100\%) fez o uso da TARV. Tais achados se repetiram na variável evolução do tratamento, haja vista que a maioria dos homens $(78,95 \%)$ e a totalidade das mulheres (100,00 \%) estavam continuidade de tratamento do HIV/AIDS (TABELA 4).

Tabela 1- Distribuição da faixa etária e do estado civil dos idosos portadores HIV/AIDS de acordo com o sexo.

\begin{tabular}{|c|c|c|c|c|c|c|}
\hline \multirow{3}{*}{ VARIÁVEIS } & \multicolumn{6}{|c|}{ SEXO } \\
\hline & \multicolumn{2}{|c|}{$\begin{array}{c}\text { Masculino } \\
\mathrm{n}=19\end{array}$} & \multicolumn{2}{|c|}{$\begin{array}{c}\text { Feminino } \\
n=4\end{array}$} & \multicolumn{2}{|c|}{$\begin{array}{l}\text { Total } \\
\mathbf{n}=\mathbf{2 3}\end{array}$} \\
\hline & $\mathbf{N}^{\mathbf{o}}$ & $\%$ & $\mathbf{N}^{\circ}$ & $\%$ & $\mathbf{N}^{\mathbf{0}}$ & $\%$ \\
\hline \multicolumn{7}{|l|}{ Faixa etária } \\
\hline $60-69$ anos & 18 & 94,74 & 4 & 100,00 & 22 & 95,65 \\
\hline 70-79 anos & 1 & 5,26 & 0 & 0 & 1 & 4,35 \\
\hline 80 anos ou mais & 0 & 0 & 0 & 0 & 0 & 0 \\
\hline \multicolumn{7}{|l|}{ Estado Civil } \\
\hline Solteiro (a) & 4 & 21,05 & 2 & 50,00 & 6 & 26,09 \\
\hline Casado (a) & 13 & 68,42 & 0 & 0 & 13 & 56,52 \\
\hline Viúvo (a) & 2 & 10,53 & 2 & 50,00 & 4 & 17,39 \\
\hline Divorciado (a) & 0 & 0 & 0 & 0 & 0 & 0 \\
\hline Não informado & 0 & 0 & 0 & 0 & 0 & 0 \\
\hline
\end{tabular}

Fonte: Pesquisa de campo, 2014. 
Tabela 2- Distribuição da escolaridade dos idosos portadores do HIV/AIDS de acordo com o sexo.

\begin{tabular}{|c|c|c|c|c|c|c|}
\hline \multirow{2}{*}{ VARIÁVEL } & \multicolumn{2}{|c|}{$\begin{array}{c}\text { Masculino } \\
n=19\end{array}$} & \multicolumn{2}{|c|}{$\begin{array}{c}\text { SEXO } \\
\text { Feminino } \\
\mathrm{n}=4\end{array}$} & \multicolumn{2}{|c|}{$\begin{array}{c}\text { Total } \\
\mathbf{n}=\mathbf{2 3}\end{array}$} \\
\hline & $\mathbf{N}$ & $\%$ & $\mathbf{N}$ & $\%$ & $\mathbf{N}^{\mathbf{o}}$ & $\%$ \\
\hline \multicolumn{7}{|l|}{ Escolaridade } \\
\hline Analfabeto (a) & 2 & 10,53 & 1 & 25,00 & 3 & 13,04 \\
\hline Fundamental Incompleto & 8 & 42,11 & 1 & 25,00 & 9 & 39,12 \\
\hline Fundamental Completo & 1 & 5,26 & 1 & 25,00 & 2 & 8,70 \\
\hline Médio Incompleto & 1 & 5,26 & 0 & 0 & 1 & 4,35 \\
\hline Médio Completo & 1 & 5,26 & 1 & 25,00 & 2 & 8,70 \\
\hline Superior Incompleto & 0 & 0 & 0 & 0 & 0 & 0 \\
\hline Superior Completo & 2 & 10,53 & 0 & 0 & 2 & 8,70 \\
\hline Não informado & 4 & 21,05 & 0 & 0 & 4 & 17,39 \\
\hline
\end{tabular}

Fonte: Pesquisa de campo, 2014.

Tabela 3- Distribuição da procedência e do acometimento de coinfecção dos idosos portadores do HIV/AIDS de acordo com o sexo.

\begin{tabular}{|c|c|c|c|c|c|c|}
\hline \multirow{2}{*}{ VARIÁVEIS } & \multicolumn{2}{|c|}{$\begin{array}{c}\text { Masculino } \\
\mathbf{n}=19\end{array}$} & \multicolumn{2}{|c|}{$\begin{array}{c}\text { Feminino } \\
n=4\end{array}$} & \multicolumn{2}{|c|}{$\begin{array}{c}\text { Total } \\
\mathbf{n}=\mathbf{2 3}\end{array}$} \\
\hline & $\mathbf{N}$ & $\%$ & $\mathbf{N}$ & $\%$ & $\mathbf{N}^{\mathbf{o}}$ & $\%$ \\
\hline \multicolumn{7}{|l|}{ Procedência } \\
\hline Belém ou Região Metropolitana & 13 & 68,42 & 4 & 100,00 & 17 & 73,91 \\
\hline Interior & 6 & 31,58 & 0 & 0 & 6 & 26,09 \\
\hline Não informado & 0 & 0 & 0 & 0 & 0 & 0 \\
\hline \multicolumn{7}{|l|}{ Coinfecção acometida } \\
\hline Nenhuma & 11 & 57,91 & 3 & 75,00 & 14 & 60,86 \\
\hline Tuberculose & 4 & 21,05 & 0 & 0 & 4 & 17,39 \\
\hline Herpes & 1 & 5,26 & 0 & 0 & 1 & 4,35 \\
\hline Pneumonia & 1 & 5,26 & 1 & 25,00 & 2 & 8,70 \\
\hline Neurotoxoplasmose & 0 & 00,00 & 0 & 0 & 0 & 0 \\
\hline Toxoplasmose & 0 & 00,00 & 0 & 0 & 0 & 0 \\
\hline Hepatite C & 1 & 5,26 & 0 & 0 & 1 & 4,35 \\
\hline Linfoma de MALT & 1 & 5,26 & 0 & 0 & 1 & 4,35 \\
\hline
\end{tabular}

Fonte: Pesquisa de campo, 2014. 
Tabela 4- Distribuição do uso de Terapia antirretroviral (TARV) e da evolução do tratamento dos idosos portadores do HIV/AIDS de acordo com o sexo.

\begin{tabular}{|c|c|c|c|c|c|c|}
\hline \multirow{2}{*}{ VARIÁVEIS } & \multicolumn{2}{|c|}{$\begin{array}{c}\text { Masculino } \\
\mathbf{n}=19\end{array}$} & \multicolumn{2}{|c|}{$\begin{array}{c}\text { SEXO } \\
\text { Feminino } \\
\mathrm{n}=4\end{array}$} & \multicolumn{2}{|c|}{$\begin{array}{c}\text { Total } \\
\mathbf{n}=\mathbf{2 3}\end{array}$} \\
\hline & $\mathbf{N}$ & $\%$ & $\mathbf{N}$ & $\%$ & $\mathbf{N}^{\mathbf{o}}$ & $\%$ \\
\hline \multicolumn{7}{|l|}{ Uso de TARV } \\
\hline Sim & 17 & 89,48 & 4 & 100,00 & 21 & 91,30 \\
\hline Não & 1 & 5,26 & 0 & 0 & 1 & 4,35 \\
\hline Não informado & 1 & 5,26 & 0 & 0 & 1 & 4,35 \\
\hline \multicolumn{7}{|l|}{ Evolução do tratamento } \\
\hline Continuidade & 15 & 78,95 & 4 & 100,00 & 19 & 82,61 \\
\hline Óbito & 3 & 15,79 & 0 & 0 & 3 & 13,04 \\
\hline Abandono & 1 & 5,26 & 0 & 0 & 1 & 4,35 \\
\hline Transferência & 0 & 0 & 0 & 0 & 0 & 0 \\
\hline Não informado & 1 & 5,26 & 0 & 0 & 1 & 4,35 \\
\hline
\end{tabular}

Fonte: Pesquisa de campo, 2014.

\section{DISCUSSÃO}

Dos 1.134 prontuários analisados, apenas $23(2,03 \%)$ eram de idosos (idade igual ou superior a 60 anos). Na análise de Oliveira, Paz e Melo (2013), dos 4.258 casos novos no Distrito Federal, 89 (2,09\%) eram idosos. Embora a porcentagem seja pequena, o crescimento anual foi contínuo.

Nesta pesquisa, observou-se que os idosos mais infectados pelo HIV/AIDS foram os homens $(82,61 \%)$ em relação às mulheres $(17,39 \%)$. No estudo de Ultramari et al (2011), que após a análise de 208 prontuários de indivíduos portadores do HIV/AIDS com idade de 50 anos ou mais, encontrou-se que $95(45,70 \%)$ eram do sexo feminino e 113 $(54,30 \%)$ do sexo masculino. Essa tendência, também, foi vista no estudo de Toledo et al
(2010), acometendo mais os homens $(62,40 \%)$ que as mulheres $(37,60 \%)$.

Essa maior incidência nos homens com mais de 60 anos pode ser explicada pela permanência de algumas questões culturais como a aceitação social da infidelidade e da multiplicidade de parceiras, além da não prática do sexo seguro, pois isso nunca fez parte da vida dos mesmos (FONTES; SALDANHA; ARAÚJO, 2007).

Em relação à incidência na pesquisa de Oliveira, Paz e Melo (2013), embora a incidência de AIDS em idosos tenha apresentado um menor coeficiente quando comparada as demais faixas etárias, conferese uma nova realidade à epidemia, especialmente, quando observada a faixa de 
60 a 69 anos de idade, sendo esse intervalo de idade o mais encontrado neste estudo $(95,65 \%)$.

Dos anos de 2000 a 2013, de acordo com o Sistema de Informática do SUS (DATASUS), 399 casos de AIDS foram identificados no Pará em indivíduos com 60 anos ou mais (BRASIL, 2013).

Um importante indicador para a elevação das taxas de idosos infectados pelo HIV/AIDS é a baixa escolaridade, haja vista que indivíduos com menos tempo de estudo tendem a assimilar as informações de maneira inadequada e contribui para uma pobre compreensão da doença, embora o indivíduo receba informações corretas e de fontes seguras (SILVA et al, 2011; WONG; CARVALHO, 2006).

Quando analisadas as características epidemiológicas, no estudo de Silva et al (2011), a baixa escolaridade foi evidente, englobando $86,90 \%$ dos pacientes idosos, quando somados os percentuais de analfabetos a ensino fundamental, aproximando-se dos valores encontrados nessa pesquisa, que apresentou 60,86\% de baixa escolaridade.

No presente estudo a maioria dos idosos do sexo masculino $(68,42 \%)$ assim como a totalidade do sexo feminino $(100,00 \%)$ tinham Belém (capital) ou região metropolitana como procedência. No estudo de Sousa, Suassuna e Costa (2009) realizado em João Pessoa-PB, por outro lado, o interior foi o de maior porcentagem tanto nos homens $(90,91 \%)$ quanto nas mulheres $(88,88 \%)$.

Um dos maiores desafios para o sucesso do tratamento HIV/AIDS é o uso da terapia antirretroviral (TARV). $\mathrm{O}$ uso da TARV prolonga e melhora a qualidade de vida, diminui episódios mórbidos e o número de internações (SILVA; WALDMAN; MARCON, 2009). Na pesquisa de Padoin et al (2013), dos 72 pacientes com idade acima de 50 anos, 51 (70,83\%) faziam uso de TARV e $21(29,17 \%)$ não faziam, aproximando-se tais valores deste estudo: $91,30 \%$ fizeram uso de TARV, de acordo com os dados nos prontuários da URE DIPE.

O uso da TARV em conjunto com a generalização das profilaxias primária e secundária levaram a uma redução apreciável do número de algumas infecções oportunistas (FAGUNDES et al, 2010). Neste estudo, a maioria dos idosos $(60,86 \%)$ não adquiriu coinfecção paralela ao HIV/AIDS.

Levando-se em consideração a evolução de tratamento, notou-se que $82,61 \%$ estavam em continuidade e que 13,04\% 
evoluíram a óbito, fato que se assemelha ao estudo de Vieira, Alves e Sousa (2014), no qual $76 \%$ encontravam-se vivos e $21 \%$ vieram a óbito.

Essa maior continuidade no tratamento e menos óbitos, pode ser relacionada ao uso da TARV que reduz a ocorrência de doenças oportunistas, além de aumentar significativamente o tempo de sobrevida (SILVA; WALDMAN; MARCON, 2009).

\section{CONCLUSÃO}

Nesta pesquisa clínica e epidemiológica, observou-se a maioria dos idosos eram do sexo masculino, na faixa etária de 60-69 anos, casados, tinha escolaridade de ensino fundamental incompleto, era de Belém ou região metropolitana, não adquiriu nenhuma coinfecção-HIV/AIDS, fez uso de TARV e estava em continuidade de tratamento. Acredita-se que essa amostra seja maior do que a que foi apresentada, em virtude dos aumentos do número de idosos e da expectativa de vida populacional, das subnotificações e dos "não-diagnósticos" em idosos portadores do HIV/AIDS, assim como a baixa procura do idoso pelos serviços de saúde especializados. Logo, sugere-se que novos estudos sejam realizados, abordando uma amostra maior.

\section{REFERÊNCIAS}

BATISTA, A.F.O. et al. Idosos: Associação entre o conhecimento da aids, atividade sexual e condições sociodemográficas.

\section{Revista Brasileira de Geriatria e}

Gerontologia, Rio de Janeiro, v.14, n.1, p.3948, 2011. Disponível em:

$<$ http://revista.unati.uerj.br/pdf/rbgg/v14n1/v1 4n1a05.pdf>. Acesso em: 17 set. 2014.

BERTONI, R.F. et al. Perfil demográfico e socioeconômico dos portadores de HIV/AIDS do Ambulatório de Controle de DST/AIDS de São José, SC. Arquivos Catarinenses de Medicina, v. 39, n. 4, 2010. Disponível em: $<$ http://www.acm.org.br/revista/pdf/artigos/83 5.pdf>. Acesso em: 16 set. 2014.

BRASIL. Ministério da Saúde. Serviço de Vigilância em Saúde. Departamento de DST/ AIDS e Hepatites Virais. Boletim Epidemiológico Aids - DST. Brasília: Ministério da Saúde, 2009 Disponível em: <http://www.aids.gov.br/sites/default/files/pu blicacao/2009/boletim2009_final_pdf_24513. pdf>. Acesso em: 17 set. 2013.

BRASIL. Ministério da Saúde. Departamento de Informática do SUS. Casos de aids 


\section{identificados no Pará Frequência por Ano}

Diagnóstico segundo Faixa etária, de 2000-

2013. Ministério da Saúde, jul. 2013.

Disponível em:

<http://www2.aids.gov.br/cgi/tabcgi.exe?tabn et/pa.def>. Acesso em: 23 nov. 2014.

BRASIL. Ministério da Saúde. Serviço de Vigilância em Saúde. Secretaria de Vigilância em Saúde - Departamento de DST, Aids e Hepatites Virais. Boletim Epidemiológico HIV - AIDS. Brasília: Ministério da Saúde, ano III, n. 1, 2014. Disponível em: < http://www.aids.gov.br/sites/default/files/anexos/p ublicacao/2014/56677/boletim 2014_1_pdf 6025 4.pdf >. Acesso em: 02 dez. 2014. CAMBRUZZI, C.; LARA, G.M. HIV/AIDS em idosos brasileiros. Revista Conhecimento Online, v.1, n. 4, 2012. Disponível em: <http://www.feevale.br/site/files/documentos/ pdf/58664.pdf>. Acesso em: 16 nov. de 2014.

FAGUNDES, V.H.V. et al. Infecções oportunistas em indivíduos com infecção pelo HIV e relação com uso de terapia antirretroviral. Acta Scientiarum. Health Sciences, Maringá, v. 32, n. 2, p. 141-145, 2010. Disponível em:

<http://periodicos.uem.br/ojs/index.php/ActaS ciHealthSci/article/viewFile/4508/4508>.

Acesso em: 27 nov. 2014.
FONTES, K.S.; SALDANHA, A.A.W.; ARAÚJO, L.F. Representações do HIV na terceira idade e a vulnerabilidade no idoso. ANAIS: 7 Virtual Congress HIV-AIDS , maio, 2007. Disponível em:

<file:///G:/revista/LIVRO\%20VII\%20CONG RESSO \%20VIRTUAL\%20HIV\%20AIDS\% 2 0o\%20hiv\%20aids\%20na\%20crian\%C3\%A7 a\%20e\%20no\%20idoso.pdf >. Acesso em: 30 nov. 2014.

GARCIA, G.S. et al. Vulnerabilidade dos Idosos frente ao HIV/Aids: Tendências da Produção Científica Atual no Brasil. Jornal

\section{Brasileiro de Doenças Sexualmente}

Transmissíveis, v. 24, n. 13, 2012.

Disponível em:

<http://www.dst.uff.br/revista24-3-2012/7-

Vulnerabilidade_idosos_aids.pdf $>$. Acesso em: 10 out. 2014.

OLIVEIRA, M.L.C.; PAZ, L.C.; MELO, G.F. Dez anos de epidemia do HIV-AIDS em maiores de 60 anos no Distrito Federal-Brasil. Revista Brasileira de Epidemiologia, v.16, n.1, p.30-39, 2013. Disponível em: <http://www.scielo.br/pdf/rbepid/v16n1/1415 -790X-rbepid-16-01-0030.pdf >. Acesso: 14 nov. 2014.

PADOIN, S.M.M. et al. Terapia antirretroviral da AIDS em adultos acima de 50 anos: prevalência e classificação de não aderentes. Revista Enfermería Glogal, n. 31, 
jul. 2013. Disponível em:

<http://revistas.um.es/eglobal/article/viewFile leglobal.12.3.151521/149881>. Acesso e: 27 nov. 2014.

SERRA, A. et al. Percepção de vida dos idosos portadores do HIV/AIDS atendidos em centro de referência estadual. Revista Saúde em Debate, Rio de Janeiro, v. 37, n. 97, abr./jun. 2013. Disponível em:

<http://www.scielo.br/pdf/sdeb/v37n97/v37n9 7a11.pdf>. Acesso em: 18 nov. 2014.

SILVA, H.R. et al. Características clínicoepidemiológicas de pacientes idosos com aids em hospital de referência, Teresina-PI, 1996 a 2009. Revista Epidemiologia e Serviços de Saúde, Brasília, v. 20, n. 4, p.499-507, out./dez. 2011. Disponível em:

<http://scielo.iec.pa.gov.br/pdf/ess/v20n4/v20 n4a09.pdf >. Acesso em: 25 nov. 2014.

SILVA, A.L.C.N.; WALDMAN, M.A.P.; MARCON, S.S. Adesão e não-adesão à terapia anti-retroviral: as duas faces de uma mesma vivência. Revista Brasileira de

Enfermagem, Brasília, v.62, n. 2, p. 213-220, mar./abr. 2009. Disponível em: $<$ http://www.scielo.br/pdf/reben/v62n2/a07v6 2n2.pdf>. Acesso em: 20 nov. 2014.

SILVEIRA, M.M. et al. Sexualidade e Envelhecimento: discussões sobre a AIDS.
Revista Temática Kairós Gerontologia, São

Paulo, v.14, n.5, 2011. Disponível em:

$<$ http://revistas.pucsp.br/index.php/kairos/arti cle/download/5673/7347>. Acesso em: 17 nov. 2014.

SOUSA, C.S.O; SILVA, A.L. O cuidado a pessoas com HIV/aids na perspectiva de profissionais de saúde. Revista da Escola de Enfermagem da USP, v. 47, n. 4, 2013.

Disponível em:

<http://www.scielo.br/pdf/reeusp/v47n4/0080 -6234-reeusp-47-4-0907.pdf>. Acesso em: 16 nov. 2014.

SOUSA, A.C.A.; SUASSUNA, D.S.B.;

COSTA, S.M.L. Perfil clínico-epidemiológico de idosos com AIDS. Jornal Brasileiro de Doenças Sexualmente Transmissíveis, v. 21, n. 1, p. 22-26, 2009. Disponível em: <http://www.dst.uff.br/revista21-1-2009/5Perfil\%20Clinico-Epidemiologico\%20JBDST\%2021(1)\%202009.pdf >. Acesso em: 17 nov. 2014.

TOLEDO, L.S.G. et al. Características e tendência da AIDS entre idosos no Estado do Espírito Santo. Revista da Sociedade Brasileira de Medicina Tropical, v. 43, n.3, mai./jun. 2010. Disponível em: $<$ http://www.scielo.br/pdf/rsbmt/v43n3/10.pdf >. Acesso em: 16 nov. 2013. 
ULTRAMARI, L. et al. Perfil clínico e

epidemiológico da infecção pelo HIV/aids em idosos. Revista Eletrônica de Enfermagem, v.13, n.3, p. 405-412, jul./set. 2011.

Disponível em:

<http://www.revistas.ufg.br/index.php/fen/arti cle/view/11816/10641 >. Acesso: 20 nov. 2014.

VIEIRA, G.D.; ALVES, T.C.; SOUSA, C.M.

Perfil da AIDS em indivíduos acima de 50 anos na região amazônica. Revista Brasileira de Geriatia e Gerontoogia, Rio de Janeiro, v. 17, n. 1, p. 61-66, 2014. Disponível: $<$ http://www.scielo.br/pdf/rbgg/v17n1/18099823-rbgg-17-01-00061.pdf >. Acesso: 15 nov. 2014.

WONG, L.L.R.; CARVALHO, J.A. O rápido processo de envelhecimento populacional do Brasil: sérios desafios para as políticas públicas*. Revista Brasileira de Estudos de População, São Paulo, v. 23, n. 1, p. 5-26, jan./jun. 2006. Disponível em: <http://www.scielo.br/pdf/rbepop/v22n2/v22n $\underline{2 \mathrm{a} 01}$ >. Acesso em: 14 nov. 2014. 